\title{
Low Temperature Electron Microscopy and Manipulation of Electronic Order
}

\author{
Ismail El Baggari ${ }^{1}$, David Baek ${ }^{1}$, Michael Zachman ${ }^{2}$ and Lena Kourkoutis ${ }^{1}$
}

${ }^{1}$ Cornell University, Ithaca, New York, United States, ${ }^{2}$ Oak Ridge National Laboratory, Oak Ridge, Tennessee, United States

Scanning transmission electron microscopy (STEM) affords atomic-scale visualizations of the structure of materials, but achieving a deeper understanding requires coupling imaging to a relevant in situ stimulus. For instance, the majority of so-called quantum materials exhibit exotic properties --spanning superconductivity, charge and orbital order, multiferroic order, and metal-insulator transitions-exclusively below room temperature, which necessitates cryogenic imaging capabilities. Recent demonstrations of cryogenic STEM with high resolution, signal-to-noise ratio (SNR), and precision open the door for understanding said exotic electronic and structural phases $[1,2,3]$. Here, we illustrate the power of high-resolution cryogenic STEM for observing and mapping low temperature electronic phenomena.

High-angle annular dark-field (HAADF) STEM measurements are performed in an aberration-corrected FEI Titan Themis, equipped with a cryo-box to prevent ice build-up, operating at $300 \mathrm{kV}$ for radiationhard materials (oxides) and $80 \mathrm{kV}$ or $120 \mathrm{kV}$ for radiation-sensitive materials. Low temperature measurements use a double-tilt side-entry cryogenic specimen holder (Gatan Model 636). Stable imaging conditions were obtained after allowing the liquid nitrogen cooled holder to settle inside the microscope for $\sim 2$ hours. Stacks of fast-acquisition images (0.5-1 $\mu \mathrm{s} / \mathrm{px})$ are acquired and aligned using rigid registration optimized for low SNR data, to mitigate the drift instabilities introduced by cryogenic cooling [2]. Figure 1a shows a high-resolution HAADF STEM image of the layered material TaTe 2 at $\sim 93 \mathrm{~K}$. The information transfer determined from the Fourier transform (Fig. 1b) is $0.64 \AA$, approaching the imaging performance more routinely achieved at room temperature.

In a first application, we discuss the low temperature ordered phase of $\mathrm{Nd}_{1 / 2} \mathrm{Sr}_{1 / 2} \mathrm{MnO}_{3}$. A cryogenic HAADF STEM image and its Fourier transform are shown in Figs. 1c and d, respectively. In the image, the $\mathrm{Nd} / \mathrm{Sr}$ atomic columns (bright) and $\mathrm{Mn}$ columns (dim) are well resolved. Compared to room temperature data, no changes are immediately visible in real space, however, the Fourier transform shows additional superlattice peaks (blue circles). These indicate that a structural transition has taken place below room temperature, inducing a doubled unit cell. The cryogenic data not only exhibits high resolution but also high SNR, which enables direct maps of picometer-scale lattice distortions in the low temperature phase and a deeper understanding of the nature of the transition. Figure 1c shows a map of periodic lattice displacements (PLD) at $93 \mathrm{~K}$, where a twofold superlattice emerges from the alternation of transverse distortions of the cations. A long-standing controversy about this material concerns the question of whether the PLD is centered on the sites (Mn) or on the bonds (oxygen ligand) [4,5]. The map shown in Fig. 2e is consistent with the former proposition. However, we also observe an alternative displacement pattern in a different region (Fig. 1f), consistent with a state mixing site- and bond-centered order. Thus, the demonstrated high-resolution cryogenic capabilities provide a powerful approach to understand low temperature electronic phenomena at the local scale, through maps of the picoscale lattice responses across phase transitions.

Beyond access to low temperature phenomena, an even more exciting prospect is the utilization of in situ temperature cycles to not only visualize, but also manipulate emergent electronic phenomena at the atomic 
scale. As an illustration, we study the layered charge density wave (CDW) material 1T-TaS2 which exhibits a nearly-commensurate CDW (NCCDW) below $350 \mathrm{~K}$ and a commensurate CDW (CCDW) below $160 \mathrm{~K}$. Figure 1a shows a room-temperature STEM image of 1T-TaS 2 which contains NCCDW twins that were induced externally via light excitation (Fig. 2b, red/blue triangles) [6]. Local Fourier transforms (Fig. 2c) show that individual twins are spatially separated. By mapping the local amplitude of each twin $(A=+1$ for twin-1, $A=-1$ for twin-2) over a large field-of-view, we visualize the CDW domain morphology and boundary (Fig. 2d). To manipulate the boundary, we cool the sample through the firstorder NCCDW-CCDW transition and observe that the twins undergo a significant rearrangement: they become overlapping ( $A$ closer to 0 ) and no clear boundary separates them (Fig. 2e). Upon warming back to room temperature, the twins in the same region as Fig. $2 \mathrm{~d}$ become spatially separated again but with a completely different domain morphology (Fig. 2f), paving the way for in situ visualization and manipulation of exotic electronic order. [7]
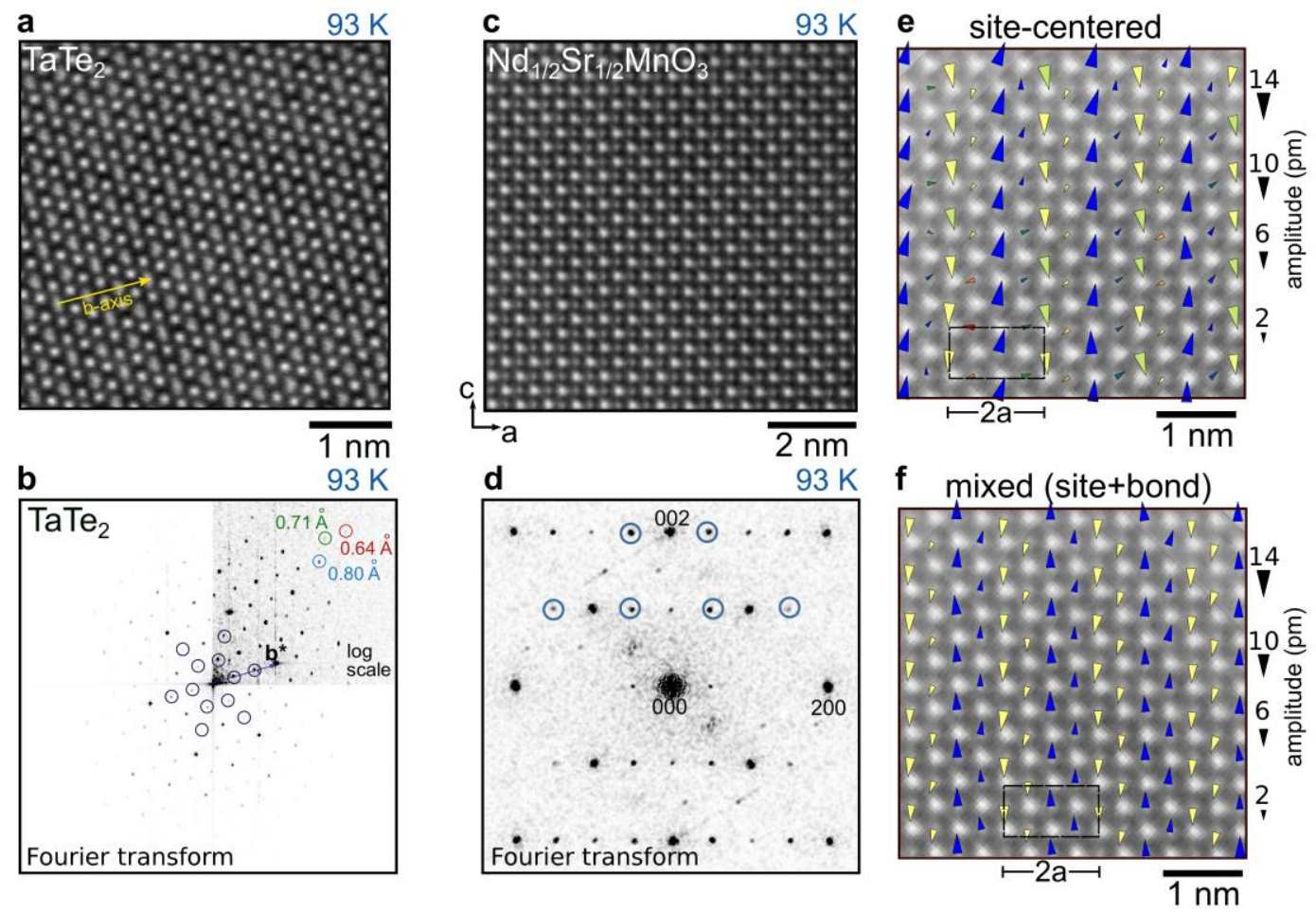

Figure 1. Cryogenic STEM mapping of picoscale lattice order. a. Cryogenic HAADF-STEM image of TaTe2 showing high resolution and signal-to-noise ratio. b. Fourier transform showing information transfer up to 0.64 Angstrom. c. Cryogenic HAADF-STEM image of Nd1/2Sr1/2MnO3 at $93 \mathrm{~K}$. The $\mathrm{Nd} / \mathrm{Sr}$ (bright) and $\mathrm{Mn}(\mathrm{dim})$ atomic columns are clearly resolved. d. Fourier transform of the HAADFSTEM image showing the presence of superlattice peaks (blue circles). These indicate the presence of a twofold supercell. e. Map of picoscale periodic lattice displacements (PLD) indicating site-centered order. The displacements have transverse polarization. f. A different PLD pattern characteristic of mixed (site+bond) order is observed in a different region of the same sample suggesting coexistence of these two ordered states. 

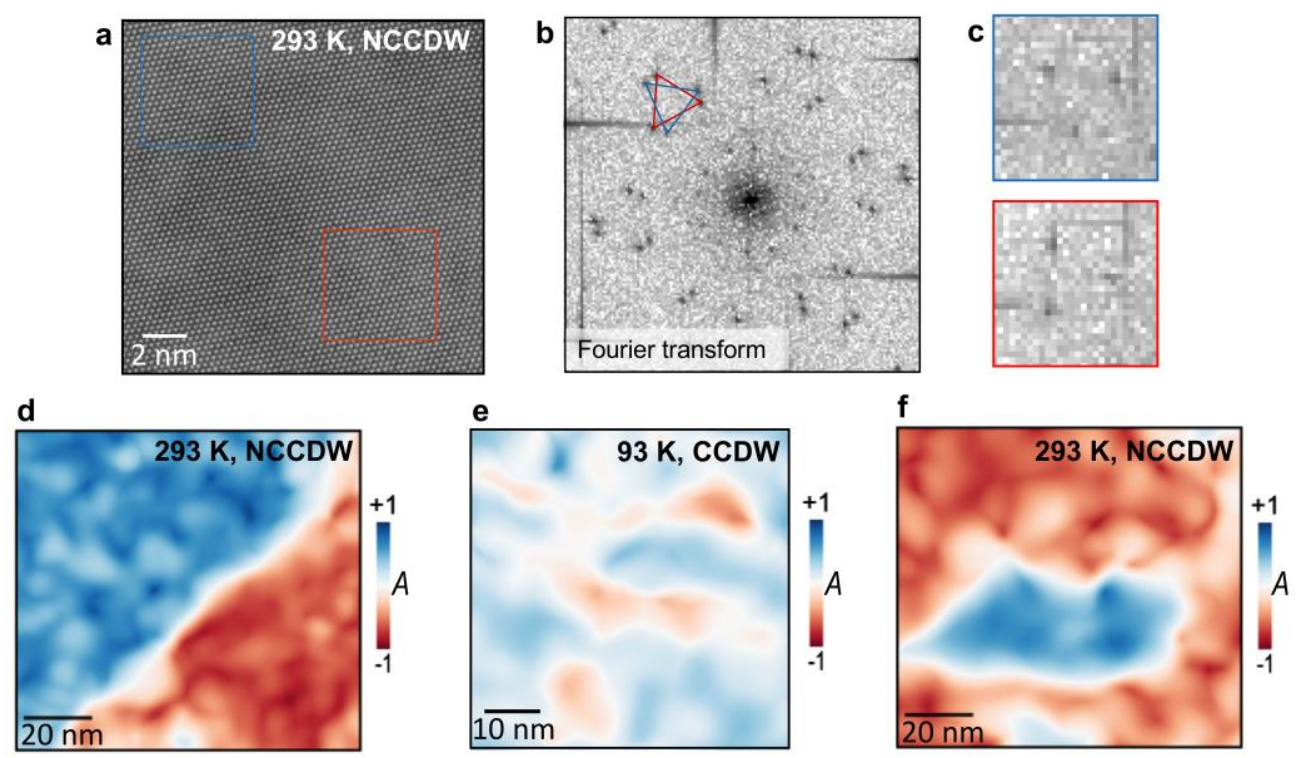

Figure 2. Temperature cycle effect on charge density wave states a. Room-temperature HAADF-STEM image of 1T-TaS2. b. Fourier transform of the HAADF-STEM image shows the presence of NCCDW twins (blue/red triangle). c. Local Fourier transforms (see regions in a) indicate that the twins are spatially separated. d. Map of the local amplitude ( $A=+1 /-1$ for twin-1/twin-2) shows a domain boundary separating the twins. e. Cooling into the CCDW phase, the twins become more overlapping with A closer to 0. $\mathrm{f}$. Warming back to room temperature, we recover the spatially separated NCCDW twins but with a different morphology (in the same region shown in d).

References

[1] I. El Baggari, et al., Proc. Natl. Acad. Sci. 115 (2018), p. 1445-1450.

[2] B. H. Savitzky, et al., Ultramicroscopy 191 (2018), p. 56-65.

[3] B. H. Goodge, et al., arXiv:2001.11581.

[4] D.V. Efremov, et al., Nature Materials 3 (2004), p. 853-856.

[5] P. G. Radaelli, et al., Phys. Rev. B 59 (1999), p. 14440.

[6] A. Zong, et al., Science Advances 4 (2018), eaau550.

[7] This work was supported by AFOSR (FA 9550-16-1-0305) and NSF (DMR-1539918, DMR-1429155, DMR-1719875). We acknowledge Di Lu, Yasuyuki Kawasaki, Elizabeth A. Nowadnick, Harold Hwang, Anshul Kogar, Alfred Zong and Nuh Gedik for their contribution. 